\title{
Diagnóstico de enfermagem risco de infecção e infecções relacionadas à assistência à saúde em pacientes com aids
}

Nursing diagnosis risk for infection and cross-infections in aids patients

Diagnóstico de enfermería Riesgo de Infección e Infecciones Asociadas

a la Atención de Salud en pacientes con sida

Cristiane da Câmara Marques ${ }^{1}$ (D) https://orcid.org//0000-0002-9328-4908

Vanessa Pinheiro Barreto ${ }^{1}$ enttps://orcid.org//0000-0002-9205-7850

Bárbara Coeli Oliveira da Silva ${ }^{1}$ e https://orcid.org/0000-0002-2933-0930

Marina Marisa Palhano dos Santos ${ }^{1}$ @ https://orcid.org/0000-0003-3536-3728

Eliabe Rodrigues de Medeiros ${ }^{1}$ (D) nttps://orcid.org/0000-0002-8755-238X

Gabriela Souza Damásio Guedes ${ }^{1}$ Đ https://orcid.org/0000-0002-2854-9543

Elaine de Souza Martins ${ }^{1}$ id hitps://orcid.org/0000-0001-7329-3025

Alexsandra Rodrigues Feijãa ${ }^{1}$ io nttps://orcid.org/0000-0002-8686-9502

Como citar:

Marques CC, Barreto VP, Silva BC, Santos MM,

Medeiros ER, Guedes GS, et al. Diagnóstico

de enfermagem risco de infecção e infecções relacionadas à assistência à saúde em pacientes com aids. Acta Paul Enferm. 2021;34:eAPE00365.

DOI

http://dx.doi.org/10.37689/actaape/2021A000365

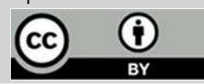

Diagnósticos de enfermagem; Síndrome de imunodeficiência adquirida; Fatores de risco;

Infecção hospitalar

Keywords

Nursing diagnosis; Acquired immunodeficiency syndrome; Risk factors; Cross infection

Descriptores

Diagnóstico de enfermería; Síndrome de inmunodeficiencia adquirida; Fatores de riesgo Infección hospitalaria

Submetido

28 de fevereiro de 2020

Aceito

2 de dezembro de 2020

Autor correspondente

Alexsandra Rodrigues Feijão E-mail: alexsandrarf@hotmail.com

\section{Resumo}

Objetivo: Identificar quais fatores de risco do diagnóstico de enfermagem risco de infecção estão associados a chances maiores de pessoas com HIV/aids hospitalizadas desenvolverem Infecções Relacionadas à Assistência à Saúde.

Métodos: Estudo caso-controle no qual os casos foram pacientes com aids hospitalizados que apresentaram Infecções Relacionadas à Assistência à Saúde $(n=104)$ e os controles foram pacientes com aids hospitalizados que não evoluíram para Infecções Relacionadas à Assistência à Saúde $(n=104)$. Usaram-se 0 teste quiquadrado de Pearson e a regressão logística, e calculou-se a odds ratio.

Resultados: Peristaltismo alterado, tabagismo, nível reduzido de hemoglobina e leucopenia foram significativamente associados com o desfecho estudado. Na regressão logística, a redução na hemoglobina foi considerada um fator preditor da detecção de risco de infecção.

Conclusão: Os indicadores tabagismo, leucopenia e nível reduzido de hemoglobina foram identificados na regressão como os preditores mais importantes para identificar o risco de infecção em pessoas vivendo com HIV/aids.

\section{Abstract}

Objective: To identify which risk factors of the Nursing Diagnosis Risk of infection are associated with a greater chance of Hospitalized People Living with HIV/AIDS developing Healthcare-associated Infections.

Methods: This is a case-control study in which the cases were composed by hospitalized AIDS patients who presented Healthcare-associated Infections $(n=104)$ and, the controls by those who did not progress to Healthcare-associated Infections $(n=104)$. The Pearson Chi-square test, Odds Ratio calculations for risk factors and Logistic Regression were used.

Results: Altered peristalsis, smoking, decreased hemoglobin and leukopenia were significantly associated with the outcome investigated. In logistic regression, the decrease in hemoglobin was considered a predictor factor for the detection of infection risk.

Conclusion: The indicators smoking, leucopenia and decreased hemoglobin were recognized in the regression as the most important predictors for identifying the risk of infection in People Living with HIV/AIDS.

\section{Resumen}

Objetivo: Identificar qué factores de riesgo del diagnóstico de enfermería Riesgo de Infección están relacionados con mayores probabilidades de que personas con el VIH/sida hospitalizadas presenten Infecciones Asociadas a la Atención de Salud. 
Métodos: Estudio caso-control, en el cual los casos fueron pacientes con sida hospitalizados que presentaron Infecciones Asociadas a la Atención de Salud $(n=104)$ y los controles fueron pacientes con sida hospitalizados que no contrajeron Infecciones Asociadas a la Atención de Salud ( $n=104)$. Se utilizó la prueba $\chi^{2}$ de Pearson y la regresión logística y se calculó el odds ratio.

Resultados: El peristaltismo alterado, el tabaquismo, el nivel reducido de hemoglobina y la leucopenia fueron significativamente asociados al resultado estudiado. En la regresión logística, la reducción de la hemoglobina fue considerada un factor predictor de la detección del riesgo de infección.

Conclusión: Los indicadores tabaquismo, leucopenia y nivel reducido de hemoglobina fueron identificados en la regresión como los predictores más importantes para identificar el riesgo de infección en personas que viven con el VIH/sida.

\section{Introdução}

Após quase quarenta anos do início da epidemia da síndrome da imunodeficiência adquirida (aids), ela ainda é um grave problema de saúde no mundo todo. ${ }^{(1)}$ Entretanto, nos últimos anos, tem ocorrido uma diminuição no número de óbitos provocados pela doença (de 1,9 milhão em 2005 para 1 milhão em 2016), o que é atribuído ao uso regular da terapia antirretroviral (TARV). ${ }^{(2)}$

$\mathrm{Na}$ América Latina, essa terapia tornou-se disponível mais cedo do que em outras regióes do mundo. Apesar disso, a redução no número de óbitos foi de apenas $16 \%$, menor do que a registrada em outras regiôes, como a Ásia e o Pacífico, onde a queda foi de $39 \% .^{(2)}$ A redução na tendência de mortalidade associada à doença no Brasil é considerável, em concordância com a situação da epidemia no continente. ${ }^{(3)}$

$\mathrm{O}$ diagnóstico tardio da infecção pelo vírus da imunodeficiência humana (HIV)/aids e a falta de adesão à TARV ainda são uma realidade. Esses dois fatores favorecem o dano ao sistema imune, levando ao desenvolvimento da aids, uma vez que o indivíduo torna-se suscetível a adquirir uma variedade de comorbidades e fica vulnerável a infecções oportunistas. ${ }^{(4-6)}$

ATARV foi disponibilizada no Brasil pelo Sistema Único de Saúde na década de 1990, e o acesso a esses medicamentos trouxe uma série de benefícios para as pessoas vivendo com HIV/aids (PVHA), incluindo maior sobrevida e melhor qualidade de vida, reduzindo, portanto, a disseminação da epidemia. ${ }^{(7)}$

Entretanto, efeitos colaterais e complicaçóes associados ao uso prolongado da TARV têm sido observados, como o aumento progressivo da incidência de doenças não infecciosas como o principal problema de saúde nessa população. Atualmente, problemas metabólicos como dislipidemia, resistência à insulina, aumento da gordura intra-abdominal e redução da gordura periférica e doenças cardio- vasculares e neoplásicas estáo liderando as causas de óbito em pacientes com HIV/aids. ${ }^{(8,9)}$

Doenças crônicas, toxicidade à TARV de longa duração e envelhecimento são fatores que podem intensificar a necessidade de hospitalização nesses pacientes. ${ }^{(10)}$ Ela expóe os indivíduos a vários procedimentos terapêuticos para promover a melhora da condição clínica. Entretanto, a execução desses procedimentos, incluindo a inserção de cateteres venosos centrais e urinários, deixa os pacientes mais suscetíveis a desenvolver Infecçôes Relacionadas à Assistência à Saúde (IRAS) e a falecer. ${ }^{(11,12)}$

A enfermagem desempenha um papel importante na detecção precoce e na prevenção das IRAS porque é responsável por desenvolver um conjunto de açóes deliberadas e sistemáticas voltadas para reduzir a incidência e a gravidade dessas infecçóes ${ }^{(13)}$. Por esse motivo, fazer um diagnóstico acurado torna-se essencial para avaliar o risco de infecção.

Determinar o diagnóstico risco de infecção especificamente em PVHA nem sempre é uma tarefa fácil, considerando que há um senso comum de que a infecção já está estabelecida, e que, além disso, há um diagnóstico situacional. Consequentemente, os enfermeiros negligenciam o risco sob o qual o paciente está de desenvolver outras infecçôes que tenham como origem o ambiente hospitalar.

Para prevenir esse problema, existem o processo de enfermagem e a sistematização da assistência de enfermagem e suas respectivas taxonomias. Especificamente para a fase de diagnóstico, usa-se a taxonomia da NANDA-1.

Dessa forma, na tentativa de encontrar formas mais acuradas de diagnosticar o risco de infecção em PVHA durante a hospitalização, o presente estudo teve como objetivo identificar quais fatores de risco do diagnóstico de enfermagem risco de infecção estão associados com maior taxa de hospitalização de PVHA com IRAS. 


\section{Métodos}

Trata-se de estudo caso-controle retrospectivo e analítico. A fonte de casos e de controles foi o serviço de arquivos médicos e estatísticos de um hospital especializado em doenças infecciosas, toxicologia e imunobiológicos especiais localizado em Natal, Rio Grande do Norte, Brasil.

A população foram 321 pacientes com aids hospitalizados. A amostra foi selecionada em uma única instituição hospitalar para minimizar o viés de amostragem. Os critérios de inclusão foram: indivíduos com 18 anos ou mais, de ambos os sexos, com aids, hospitalizados na enfermaria e na unidade de terapia intensiva do hospital mencionado entre 2010 e 2016. Pacientes cujos prontuários náo foram identificados foram excluídos porque as informaçóes presentes nesse tipo de registro seriam usadas para complementar os dados obtidos no serviço de arquivos.

Casos e controles foram definidos a partir dos registros do Comitê de Controle de Infecção Hospitalar. Definiu-se que os casos seriam pacientes com aids hospitalizados que apresentavam IRAS e que os controles seriam pacientes com aids hospitalizados que não desenvolveram IRAS durante sua estadia no hospital.

Para obter maior acurácia estatística e considerando-se o tamanho reduzido da populaçáo, optou-se por usar a amostragem de proporção estatística ou censo. O grupo 1 (casos) incluiu todos os indivíduos que evoluíram para IRAS e cumpriam os critérios de inclusão, com 104 pacientes. Para formar o grupo 2 (controles), decidiu-se fazer um sorteio simples até se atingir um número de pacientes igual ao do grupo 1 . Ou seja, a proporção de indivíduos incluídos nos dois grupos foi 1:1.

O instrumento usado foi do tipo formulário. Ele foi elaborado de acordo com os fatores de risco do diagnóstico de enfermagem risco de infecção da NANDA-I ${ }^{(14)}$ e com a literatura que trata do assunto.

$\mathrm{O}$ formulário tinha quatro partes, sendo que elas deveriam ser preenchidas conforme a sequência preestabelecida. A primeira parte era dedicada a coletar dados sociodemográficos como idade, sexo, estado civil, escolaridade, profissão e local de origem. A segunda parte continha variáveis clínicas: ano de diagnóstico da infecção por HIV, uso de TARV, motivo da hospitalização, setor, nível de hemoglobina, nível de leucócitos, desenvolvimento de IRAS, uso de dispositivo invasivo, acesso venoso periférico, acesso venoso central, cateter urinário, cateter nasogástrico, cateter nasoenteral, traqueostomia, tubo orotraqueal e dreno. A terceira parte foi inserida para uso exclusivo com o grupo 1 (indivíduos expostos) para obter dados sobre as IRAS: tipo de material coletado para cultura, resultado do antibiograma e local da IRAS. A quarta parte continha, especificamente, os fatores de risco do diagnóstico de enfermagem risco de infecçáo da NANDA-I.

Entre os fatores de risco contemplados no diagnóstico risco de infecção, selecionaram-se aqueles que eram consistentes com a condiçáo dos pacientes e possíveis de serem coletados e medidos a partir de informaçóes extraídas de registros de pesquisa e de prontuários. As seguintes variáveis foram escolhidas: doença crônica, procedimento invasivo, defesas primárias inadequadas (pele rompida, peristaltismo alterado e tabagismo) e defesas secundárias inadequadas (nível reduzido de hemoglobina e leucopenia).

$\mathrm{O}$ instrumento foi pré-testado com $10 \%$ da amostra para que se verificasse sua adequabilidade. Houve uma dificuldade operacional na coleta dos seguintes fatores de risco: desnutriçáo, obesidade e defesas secundárias inadequadas (imunossupressáo, resposta inflamatória suprimida e vacinação inadequada). Verificou-se durante o pré-teste que esses fatores de risco não estavam disponíveis nos prontuários ou que não era possível avaliar o efeito supressor no caso dos itens imunossupressão e resposta inflamatória suprimida, o que impossibilitou a inclusão destes no formulário do estudo após sua adaptaçáo. Os itens foram reformulados para estarem de acordo com os objetivos do estudo. Os registros coletados durante a fase de pré-teste foram descartados.

Após a adequaçáo do instrumento, iniciou-se a coleta de dados, que ocorreu de abril a agosto de 2017 pela pesquisadora, seis estudantes de graduação de enfermagem, membros de um projeto de pesquisa e extensão universitária no nível de graduação e um estudante de pós-graduação em enfermagem. Registros de investigaçáo de infecção hospitalar foram solicitados para o Comitê de Controle de Infecção Hospitalar para identificar os indivíduos que cumpriam os critérios de inclusão, obter o número de identificação dos prontuários desejados, coletar os 
dados referentes às infecçóes e, numa etapa posterior, buscar os prontuários no serviço de arquivos médicos e estatísticos para adicionar informaçóes que não foram incluídas no arquivo do estudo.

Os dados foram organizados em planilhas do programa Microsoft Excel 2013. Usaram-se estatísticas descritiva e inferencial na análise de dados por meio do programa estatístico IBM SPSS Statistics, versão 20.0.

Para os dados socioeconômicos e clínicos, usou-se estatística descritiva para calcular frequências absolutas e relativas, medidas de dispersão (desvio padrão) e tendências centrais (média e mediana).

$\mathrm{Na}$ análise inferencial, o teste qui-quadrado de Pearson foi aplicado para verificar a associação entre fatores de risco de pacientes com aids nos grupos de casos e controles, e os fatores com um valor de $\mathrm{p}$ menor ou igual a 0,05 foram considerados estatisticamente significantes. Também foram calculadas as odds ratios para cada fator de risco.

Para identificar os fatores associados com o diagnóstico estudado, a regressão logística foi aplicada aos fatores de risco com significância. Usaram-se o teste de Walt para confirmar a significância dos coeficientes presentes na regressão, o teste de Hosmer-Lemeshow para avaliar a diferença entre as frequências observadas e esperadas, o teste Omnibus para verificar a significância do modelo desenvolvido e o teste Nagelkerke R2 para medir a performance global do modelo de regressão.

Depois da análise, os dados foram reunidos em tabelas que exibiam informaçóes sociodemográficas e clínicas, locais das IRAS, microrganismos encontrados nos resultados dos antibiogramas e fatores associados com a presença do diagnóstico, e a discussão fundamentada nas evidências disponíveis na literatura.

O estudo foi aprovado pelo Comitê de Ética em Pesquisa e seguiu as normas que regulamentam pesquisas com seres humanos no Brasil. A manipulação de prontuários foi autorizada por meio de um termo de consentimento assinado pelo administrador do serviço de arquivos médicos e estatísticos.

\section{Resultados}

A amostra tinha majoritariamente homens $(63,5 \%$ do grupo dos casos e $75,9 \%$ do grupo dos contro- les) e pessoas sem um parceiro ( $41,3 \%$ do grupo dos casos e $77,9 \%$ do grupo dos controles) e com uma ocupação profissional (43,3\% do grupo dos casos e $64,4 \%$ do grupo dos controles). Em relação ao local de residência, $41,3 \%$ dos casos moravam no campo e $50,9 \%$ dos controles moravam na capital do estado. Embora o estudo não tenha sido desenvolvido para uma faixa etária específica, quase toda a amostra (94,2\% dos casos e 91,3\% dos controles) foi composta de adultos com até 59 anos.

Em relação às variáveis clínicas reunidas na Tabela 1, observou-se que 36,5\% dos casos e 46,1\% dos controles foram diagnosticados com HIV entre 2007 e 2016 e que 69,2\% dos casos não usavam TARV. Enquanto 53,8\% (67,3\%) dos casos foram admitidos na unidade de terapia intensiva, 57,7\% dos controles estavam na unidade de internação, sendo que $31,7 \%$ dos casos e $21,1 \%$ dos controles foram admitidos com neurotoxoplasmose.

Tabela 1. Caracterização clínica de pacientes com aids que desenvolveram (casos) e não desenvolveram (controles) infecções associadas a cuidados de saúde

\begin{tabular}{lcc}
\hline Variáveis clínicas & Caso & Grupos \\
& $\mathbf{n}(\%)$ & Controle \\
$\mathbf{n}(\%)$
\end{tabular}


Os valores médios para hemoglobina e leucócitos foram, respectivamente, $4,84 \mathrm{~g} / \mathrm{dL}$ e $4563 / \mathrm{mm}^{3}$, conforme os resultados reunidos na Tabela 2 .

Tabela 2. Caracterização das variáveis clínicas hemoglobina e leucócitos em pacientes com aids que desenvolveram infecções associadas a cuidados de saúde

\begin{tabular}{lccccc}
\hline Variável & Média & Mediana & $\begin{array}{c}\text { Desvio } \\
\text { padrão }\end{array}$ & Mínimo & Máximo \\
\hline Hemoglobina* $^{*}$ & 4,84 & 0,00 & 5,34 & 0,00 & 27,10 \\
Leucócitos $^{*}$ & 4563,00 & 3050,00 & 5443 & 0,00 & 24600,00 \\
\hline
\end{tabular}

A Tabela 3 mostra os resultados do teste qui-quadrado de Pearson usado para avaliar a associação entre fatores de risco de pacientes com aids para os grupos casos e controles, assim como os valores da odds ratio.

Tabela 3. Caracterização dos fatores de risco para pacientes com aids que desenvolveram (casos) e que não desenvolveram (controles) infecções associadas ao cuidado de saúde

\begin{tabular}{|c|c|c|c|c|c|c|}
\hline \multirow[b]{2}{*}{ Fatores de risco } & \multicolumn{2}{|c|}{ Grupos } & \multirow{2}{*}{$\begin{array}{l}\text { Total } \\
\mathrm{n}(\%)\end{array}$} & \multirow{2}{*}{$\begin{array}{l}\text { Valor } \\
\text { de } p^{*}\end{array}$} & \multirow[b]{2}{*}{$\mathrm{OR}$} & \multirow{2}{*}{$\begin{array}{c}\text { IC de } 95 \% \\
\text { [Ll; LS] }\end{array}$} \\
\hline & $\begin{array}{l}\text { Caso } \\
\text { n(\%) }\end{array}$ & $\begin{array}{c}\text { Controle } \\
\mathrm{n}(\%)\end{array}$ & & & & \\
\hline \multicolumn{7}{|l|}{ Doença crônica } \\
\hline Presente & $85(49,4)$ & $87(50,6)$ & $172(82,7)$ & \multirow[t]{2}{*}{0,427} & \multirow[t]{2}{*}{1,144} & \multirow{2}{*}{$\begin{array}{l}{[0,557 ;} \\
2,349]\end{array}$} \\
\hline Ausente & $19(52,8)$ & $17(47,2)$ & $36(17,3)$ & & & \\
\hline \multicolumn{4}{|l|}{ Procedimento invasivo } & \multirow[t]{3}{*}{0,445} & \multirow[t]{3}{*}{2,58} & \multirow{3}{*}{$\begin{array}{l}{[0,489} \\
13,513]\end{array}$} \\
\hline Presente & $5(71,4)$ & $2(28,8)$ & $7(3,3)$ & & & \\
\hline Ausente & $99(49,3)$ & $102(50,7)$ & $201(96,6)$ & & & \\
\hline \multicolumn{7}{|l|}{$\begin{array}{l}\text { Alteração na } \\
\text { integridade da pele }\end{array}$} \\
\hline Presente & $6(50,0)$ & $6(50,0)$ & $12(5,77)$ & \multirow[t]{2}{*}{1,000} & \multirow[t]{2}{*}{1,000} & \multirow{2}{*}{$\begin{array}{l}{[0,312 ;} \\
3,208]\end{array}$} \\
\hline Ausente & $98(50,0)$ & $99(50,0)$ & $196(94,2)$ & & & \\
\hline \multicolumn{7}{|l|}{$\begin{array}{l}\text { Mudança no } \\
\text { peristaltismo }\end{array}$} \\
\hline Presente & $87(52,4)$ & $79(47,6)$ & $166(79,8)$ & \multirow[t]{2}{*}{0,167} & \multirow[t]{2}{*}{1,620} & \multirow{2}{*}{$\begin{array}{l}{[0,814} \\
3,220]\end{array}$} \\
\hline Ausente & $17(40,5)$ & $25(59,5)$ & $42(20,2)$ & & & \\
\hline \multicolumn{7}{|l|}{ Tabagismo } \\
\hline Presente & $92(55,1)$ & $75(44,9)$ & $167(80,3)$ & \multirow[t]{2}{*}{0,005} & \multirow[t]{2}{*}{2,967} & \multirow{2}{*}{$\begin{array}{l}{[1,416 ;} \\
6,211]\end{array}$} \\
\hline Ausente & $12(29,3)$ & $29(70,7)$ & $41(19,7)$ & & & \\
\hline \multicolumn{7}{|l|}{$\begin{array}{l}\text { Nível reduzido de } \\
\text { hemoglobina }\end{array}$} \\
\hline Presente & $52(80,0)$ & $13(20,0)$ & $65(31,2)$ & \multirow{2}{*}{$\begin{array}{c}< \\
0,001\end{array}$} & \multirow[t]{2}{*}{6,993} & \multirow{2}{*}{$\begin{array}{l}{[3,484} \\
14,084]\end{array}$} \\
\hline Ausente & $52(36,4)$ & $91(63,6)$ & $143(68,8)$ & & & \\
\hline \multicolumn{7}{|l|}{ Leucopenia } \\
\hline Presente & $85(57,8)$ & $62(42,2)$ & $147(70,7)$ & \multirow[t]{2}{*}{0,001} & \multirow[t]{2}{*}{3,030} & \multirow{2}{*}{$\begin{array}{l}{[1,610} \\
5,714]\end{array}$} \\
\hline Ausente & $19(31,1)$ & $42(68,9)$ & $61(29,3)$ & & & \\
\hline
\end{tabular}

*teste qui-quadrado de Pearson; $\mathrm{OR}$ - odds ratio, IC - intervalo de confiança; L- limite inferior; LS - limite superior
Com base na Tabela 3, pode-se inferir que, para um nível de significância de 5\%, a hipótese nula é rejeitada (há evidência de que existe uma associação entre tabagismo, nível reduzido de hemoglobina e leucopenia e infecçôes e, para as outras variáveis, não há evidência de associação). Fatores de risco para os quais houve evidência de associação apresentaram odds ratios relevantes, ou seja, a presença das infecçóes aumentou as chances de esses fatores também estarem presentes.

A Tabela 4 mostra a análise dos fatores de risco e a possível associação com o diagnóstico de enfermagem risco de infecção. Essa análise foi feita selecionando-se os fatores de risco que apresentaram significância estatística (tabagismo, nível reduzido de hemoglobina e leucopenia) e testando-os por meio de regressáo logística para verificar a associação combinada de fatores de risco, de modo que fosse possível expor a ocorrência do diagnóstico de enfermagem risco de infecção.

De acordo com os resultados exibidos na Tabela 4, entre os fatores de risco que apresentaram significância estatística, tabagismo, leucopenia e nível reduzido de hemoglobina foram identificados como fatores associados significativos para o diagnóstico risco de infecção. Além disso, o modelo logístico aplicado mostrou ter significância estatística de acordo com o teste Omnibus ( $\mathrm{p}<0,001)$, de modo que foi possível identificar a presença do diagnóstico estudado a partir dos três fatores de risco mencionados acima. Os resultados do teste de HosmerLemeshow indicaram significância maior do que 0,05 , o que significa que os valores previstos náo são significativamente diferentes dos observados.

O teste de Wald $(\mathrm{p}<0,05)$ mostrou que os coeficientes de todos os fatores de risco incluídos no modelo foram significantes. O coeficiente de determinação do modelo apresentou um valor de 0,267 , o que significa que os fatores incluídos no modelo de regressão explicam $26,7 \%$ da ocorrência das IRAS.

Tabela 4. Regressão logística para os fatores de risco preditores da presença do diagnóstico de enfermagem risco de infecção em pacientes com aids que desenvolveram infecções associadas aos cuidados de saúde

\begin{tabular}{|c|c|c|c|c|c|c|c|c|}
\hline Fatores de risco & Estimativa & Wald & Valor de $p$ & OR & $\begin{array}{c}\text { IC de } 95 \% \\
\text { [LI; LS] }\end{array}$ & Testes & $x^{2}$ & Valor de $p$ \\
\hline Tabagismo & 0,944 & 5,316 & 0,021 & 2,569 & {$[1,152 ; 5,731]$} & Hosmer-Lemeshow & 0,33 & 0,988 \\
\hline Leucopenia & 0,747 & 4,581 & 0,032 & 2,110 & {$[1,065 ; 4,182]$} & Omnibus & 46,563 & $<0,001$ \\
\hline Nível reduzido de hemoglobina & 1,755 & 22,857 & 0,032 & 5,785 & {$[1,77 ; 47,62]$} & Nagelkerke R2 & - & 0,267 \\
\hline
\end{tabular}

OR - odds ratio; IC - intervalo de confiança; LI - limite inferior; LS - limite superior 


\section{Discussão}

Identificou-se um número maior de homens na caracterização socioeconômica, o que corrobora o Boletim Epidemiológico HIV/aids brasileiro. ${ }^{(15)}$ Esse achado está relacionado à realidade da doença na população brasileira, na qual os homens são a maioria dos casos, fato confirmado na prática clínica, na qual se detecta um grande número de pacientes do sexo masculino vivendo com aids.

Em relação ao estado civil, houve um predomínio de pacientes que não tinham um parceiro, resultado semelhante ao reportado em outros estudos. ${ }^{(16-18)}$ Quanto à ocupaçáo profissional, a maioria dos indivíduos tinha emprego, o que corrobora achado de outro estudo. ${ }^{(19)}$

Em relação à caracterização clínica, a maioria dos pacientes que desenvolveu IRAS foi hospitalizada na unidade de terapia intensiva, o que confirmou resultados descritos em outros estudos. ${ }^{(20)}$ Esse achado está relacionado ao fato de que esse setor admite pacientes com condiçóes mais graves e imunossupressão. Além disso, a complexidade presente nesses pacientes culmina com uma maior demanda por intervençôes invasivas, uso excessivo de antibióticos e estadia prolongada no hospital, aspectos relacionados ao desenvolvimento de infecçôes.

Os controles, por sua vez, concentraram-se na enfermaria, que era destinada apenas a admissôes de PVHA. Estar hospitalizado nessa ala implica menos intervençôes, o que pode contribuir para minimizar as chances dos pacientes desenvolverem IRAS.

Ainda sobre caracterização clínica, a neurotoxoplasmose foi a principal causa de internação, seguida por pneumonia, candidíase oral, tuberculose pulmonar e diarreia. Vários estudos reportaram essas mesmas comorbidades em PVHA. ${ }^{(21-27)}$

A neurotoxoplasmose em pacientes imunossuprimidos tem se tornado a infecção oportunista mais grave em PVHA, dada a frequência, a gravidade e a dificuldade do regime terapêutico observadas em PVHA. Contagem baixa de células CD4 T e sorologia positiva para toxoplasmose são os preditores fortes dessa condiçãa. ${ }^{(28)}$

Quanto à análise dos fatores associados para o diagnóstico de enfermagem risco de infecção em pacientes de aids hospitalizados, os indicadores que compuseram o modelo de regressão logística foram: tabagismo, leucopenia e nível reduzido de hemoglobina, dado que eles foram os únicos que apresentaram significância estatística no modelo de acordo com o teste de Wald.

Um estudo mostrou que o tabagismo está associado com uma maior carga viral em pacientes com $\mathrm{HIV} / \mathrm{aids}$, e que fumantes diários eram duas vezes mais propensos a ter uma carga viral maior do que $10.000 \mathrm{cp} / \mathrm{mL}$. Esse estudo destacou que o uso do tabaco afeta a resposta à terapia antirretroviral de alta eficácia, está associado a um risco maior de rebote viral e leva a um aumento considerável no estresse oxidativo, o que poderia explicar o mecanismo responsável pela elevação da carga viral. Como consequência desse aumento da carga viral, há uma redução no número de células CD4 T, o que gera um risco maior de os pacientes serem afetados por IRAS. ${ }^{(29)}$

A leucopenia mostrou associação estatística com o desfecho estudado, aumentando a probabilidade da ocorrência dele três vezes. A leucopenia destacou-se na regressão como um fator associado para a ocorrência de IRAS. Ela está associada a uma perda considerável de células CD4 + e carga viral aumentada em PVHA. Dessa forma, a terapia antirretroviral de alta eficácia é apropriada e benéfica para reverter a mielosupressão e a redução no número de leucócitos. ${ }^{(30)}$

Um estudo mostrou que o risco de desenvolver leucopenia severa estava mais significativamente associado a pacientes que usaram TARV com zidovudina em comparaçáo com aqueles que iniciaram outro regime terapêutico de primeira linha. Como resultado da associação da TARV e da zidovudina, os pacientes apresentaram contagem baixa de leucócitos. ${ }^{(31)}$ No presente estudo, 30,7\% dos casos e 53,8\% dos controles usaram TARV, e a leucopenia observada provavelmente está relacionada a esse fato.

A queda no nível de hemoglobina foi prevalente na amostra examinada, e foi, de acordo com a regressão logística, um fator associado para a ocorrência de infecção. Esse achado corroborou o de outro estudo $^{(32)}$ que reportou que a anemia é a alteração hematológica mais comum em PVHA, e que sua prevalência é substancialmente elevada e está associada à progressão acelerada da síndrome. 
A ocorrência de anemia tem causa multifatorial, incluindo a presença de infecçóes oportunistas, deficiências nutricionais, efeito supressivo do HIV em fatores hematológicos e efeitos tóxicos de drogas antirretrovirais. ${ }^{(33)}$ No presente estudo, a redução no nível de hemoglobina pode estar relacionada à presença precoce de infecçóes oportunistas quando da admissão no hospital, ao quadro clínico desfavorável avançado e ao regime de TARV baseado em zidovudina. Um estudo ${ }^{(33)}$ mostrou que esse medicamento inibe a atividade da medula óssea, reduzindo a produção de células sanguíneas e elevando o risco de anemia.

Portanto, enfatiza-se que tabagismo, leucopenia e nível reduzido de hemoglobina são fatores que tanto isoladamente quanto em conjunto são considerados fatores associados para o risco de infecção nas pessoas avaliadas, dada a associação encontrada entre eles.

Os fatores associados podem ser usados por enfermeiros para facilitar a implementação de intervençôes de enfermagem de acordo com as necessidades de cada PVHA e, assim, ajudar a reduzir o número de complicaçóes provocadas pela progressáo e pelo tratamento da doença. ${ }^{(34)}$

Os achados descritos no presente artigo contribuem com a capacidade dos enfermeiros de fazer uma inferência diagnóstica, identificando o diagnóstico risco de infecção em pacientes com características semelhantes àquelas das pessoas da amostra. A presença desse indicador não deveria ser considerada um bom fator associado para o estabelecimento desse diagnóstico nessa população específica.

As limitaçóes do presente estudo foram: a falta de informaçôes nos prontuários, exemplificada pela ausência de resultados de testes laboratoriais; as inconsistências nos relatórios elaborados pela equipe de profissionais de saúde, incluindo a ausência do registro dos fatores de risco desnutrição, obesidade e defesas secundárias inadequadas; e a perda de documentação médica. Essa limitação impossibilitou a inclusão de alguns indivíduos na amostra. De acordo com as medidas usadas para evitar viés, a seleção de uma amostra aleatória de indivíduos que desenvolveram IRAS (casos) e que não desenvolveram IRAS (controles). Com base na estratégia para minimizar o viés de amostragem, os controles foram selecionados no hospital onde ocorreu a internação dos casos, e os participantes do estudo foram cegados para tentar reduzir o risco de viés.

\section{Conclusão}

O presente estudo verificou a elevada frequência de três indicadores clínicos na amostra examinada: procedimento invasivo, integridade da pele alterada e nível reduzido de hemoglobina. Ele também confirmou a hipótese teste de que os fatores de risco do diagnóstico de enfermagem risco de infecção são frequentes em pacientes com aids hospitalizados. Os fatores incluídos nesse diagnóstico que podem estar associados à sua ocorrência em PVHA são tabagismo, leucopenia e nível reduzido de hemoglobina, tanto isoladamente quanto em conjunto. $\mathrm{O}$ fator de risco que mostrou uma associação mais significativa com o diagnóstico foi nível reduzido de hemoglobina, tanto ao ser considerado individualmente quanto quando combinado com os outros dois fatores. Consequentemente, os enfermeiros envolvidos na prestação de cuidados para esses pacientes, ao escolher o diagnóstico em questão, deveriam considerar os fatores de risco destacados no presente estudo como indicadores para a existência de risco de infecção, de modo a determinar o diagnóstico com exatidão.

\section{Agradecimentos}

O presente estudo foi financiado pelo Conselho Nacional de Desenvolvimento Científico e Tecnológico (CNPq)/ MCTI/CNPq/Universal, protocolo 460777/2014-9.

\section{Colaborações}

Marques CC, Barreto VP, Silva BCO, Santos MMP, Medeiros ER, Guedes GSD, Martins ES e Feijāo AR declaram que contribuíram com a concepção do estudo, análise e interpretação dos dados, redação do artigo, revisão crítica relevante do conteúdo intelectual e aprovação da versão final a ser publicada. 


\section{Referências}

1. Ostadzad MH, Baroumand S, Mahmoudi MR. Optimization of Public Health Education Parameters for Controlling the Spread of HIV/AIDS Infection. Symmetry (Basel). 2020;12(659):1-12.

2. Joint United Nations Program on HIV/AIDS (UNAIDS). UNAIDS 2017 estimates. Geneva: UNAIDS; 2017.

3. Guimarães MD, Carneiro M, Abreu DM, França EB. Mortalidade por HIV/Aids no Brasil, 2000-2015: motivos para preocupação? Rev Bras Epidemiol. 2017;20(20 Suppl 1):182-90.

4. Alencar RA, Ciosak SI. Late diagnosis and vulnerabilities of the elderly living with HIV/AIDS. Rev Esc Enferm USP. 2015;49(2):229-35.

5. Silva JA, Dourado I, Brito AM, Silva CA. Factors associated with nonadherence to antiretroviral therapy in adults with AIDS in the first six months of treatment in Salvador, Bahia State, Brazil. Cad Saude Publica. 2015;31(6):1188-98.

6. Alencar RA, Ciosak SI. Aids in the elderly: reasons that lead to late diagnosis. Rev Bras Enferm. 2016;69(6):1140-6.

7. Carvalho PP, Barroso SM, Coelho HC, Penaforte FR. Factors associated with antiretroviral therapy adherence in adults: an integrative review of literature. Cien Saude Colet. 2019;24(7):2543-55.

8. Nath $\mathrm{K}$, Singh L. Cardiovascular manifestations of HIV infection- a review. J Evol Med Dent Sci. 2020;9(14):1208-14.

9. Kemal A, Teshome MS, Ahmed M, Molla M, Malik T, Mohammed J, et al. Dyslipidemia and associated factors among adult patients on antiretroviral therapy in armed force comprehensive and specialized hospital, Addis Ababa, Ethiopia. HIV AIDS (Auckl). 2020;12:221-31.

10. Mahlab-Guri K, Asher I, Bezalel-Rosenberg S, Elbirt D, Sthoeger ZM. Hospitalizations of HIV patients in a major Israeli HIV/AIDS center during the years 2000 to 2012. Medicine (Baltimore). 2017;96(18):e6812.

11. Pauletti M, Otaviano ML, Moraes AS, Schneider DS. Perfil epidemiológico dos pacientes internados em um Centro de Terapia Intensiva. Aletheia. 2017;50(1-2):38-46.

12. Menezes JM, Porto ML, Pimenta CL. Perfil da infecção bacteriana em ambiente hospitalar. Rev Ciênc Méd Biol. 2016;15(2):199-207.

13. Teymourzadeh E, Bahadori $M$, Fattahi $H$, Khodadost $M$, Shokri $A$. Nurses' competency and their role in prevention and control of hospital infections: A case study in a large military teaching hospital. Adv Hum Biol. 2019;(9):156-61.

14. Herdman TH, Kamitsuru S. Diagnósticos de enfermagem da NANDA internacionais: definições e classificação, 2015-2017. 10a ed. Porto Alegre: Art Med; 2015.

15. Brasil. Ministério da Saúde, Secretaria de Vigilância em Saúde, Departamento de Vigilância, Prevenção e Controle das Infecções Sexualmente Transmissíveis, do HIV/Aids e das Hepatites Virais. Boletim Epidemiológico - Aids e IST. Brasília (DF): Ministério da Saúde; 2017.

16. Mafra RL, Pereira ED, Varga ID, Mafra WC. Aspectos de gênero e vulnerabilidade ao HIV/aids entre usuários de dois dos Serviços de Atendimento Especializado em DST/aids de São Luís, Maranhão. Saúde Soc. 2016;25(3):641-51.

17. Okuno MF, Gosuen GC, Campanharo CR, Fram DS, Batista RE, Belasco AG. Quality of life, socioeconomic profile, knowledge and attitude toward sexuality from the perspectives of individuals living with Human Immunodeficiency Virus. Rev Lat Am Enfermagem. 2015;23(2):192-9.

18. Moura JP, Faria MR. Characterization and epidemiological profile of people living with HIV/Aids. Rev Enferm UFPE On line . 2017;11 (Supl12):5214-20.
19. Fontes MB, Crivelaro RC, Scartezini AM, Lima DD, Garcia AA, Fujioka RT. Determinant factors of knowledge, attitudes and practices regarding STD/AIDS and viral hepatitis among youths aged 18 to 29 years in Brazil. Cien Saude Colet. 2017;22(4):1343-52.

20. Souza ES, Belei RA, Carrilho CM, Matsuo T, Yamada-Ogatta SF, Andrade $\mathrm{G}$, et al. Mortality and risks related to healthcare-associated infection. Texto Contexto Enferm. 2015;24(1):220-8.

21. Pinho CM, Dâmaso BF, Gomes ET, Trajano MF, Andrade MS, Valença MP. Coping religioso e espiritual em pessoas vivendo com HIV/Aids. Rev Bras Enferm. 2017;70(2):392-9.

22. Katano H, Hishima T, Mochizuki M, Kodama Y, Oyaizu N, Ota Y, et al. The prevalence of opportunistic infections and malignancies in autopsied patients with human immunodeficiency virus infection in Japan. BMC Infect Dis. 2014;14(229):229.

23. Soares MT, Pinheiro JBF, Freitas CH, Smith AA, Moreira EF. Prevalência das coinfecções em Pacientes Notificados com Aids no Centro de Referência na Paraíba. Rev Bras Cienc Saúde. 2014;18(Supl 1):5-12.

24. Pereira AG, Matos HJ, Escosteguy CC, Marques MV, Medronho RA. Sobrevida de pacientes com Síndrome da Imunodeficiência Adquirida em hospital geral no Rio de Janeiro, a partir de dados da vigilância epidemiológica. Cad Saude Colet. 2013;21(2):160-7.

25. Álvarez Barreneche MF, Restrepo Castro CA, Hidrón Botero A, Villa Franco JP, Trompa Romero IM, Restrepo Carvajal L, et al. Hospitalization causes and outcomes in HIV patients in the late antiretroviral era in Colombia. AIDS Res Ther. 2017 Nov; 14(1):60.

26. Schuelter-Trevisol F, Pucci P, Justino AZ, Pucci N, Silva AC. Perfil epidemiológico dos pacientes com HIV atendidos no sul do Estado de Santa Catarina, Brasil, em 2010. Epidemiol Serv Saude. 2013;22(1):87-94.

27. Amorim MA, Miranda DB, Cabral RC, Batista AV. Clinical and epidemiological profile of patients with hiv/aids hospitalized at the hospital for reference in the state of Bahia, Brazil. Rev Enferm UFPE On line. 2011;5(6):1475-82.

28. Bamba S, Zoungrana J, Nikièma Z, Sondo AK, Ndiaye JL, Bretagne S. Impact of alternative treatment approach for cerebral toxoplasmosis among HIV/AIDS patients from a resource-poor setting in Burkina Faso. Ann Parasitol. 2017;63(3):173-81.

29. Pollack TM, Duong HT, Pham TT, Do CD, Colby D. Cigarette smoking is associated with high HIV viral load among adults presenting for antiretroviral therapy in Vietnam. PLoS One. 2017;12(3):e0173534.

30. Shi X, Sims MD, Hanna MM, Xie M, Gulick PG, Zheng YH, et al. Neutropenia during HIV infection: adverse consequences and remedies. Int Rev Immunol. 2014;33(6):511-36.

31. Leroi C, Balestre E, Messou E, Minga A, Sawadogo A, Drabo J, et al.; IeDEA West Africa collaboration. Incidence of Severe Neutropenia in HIV-Infected People Starting Antiretroviral Therapy in West Africa. PLoS One. 2017;12(1):e0170753.

32. Gunda DW, Kilonzo SB, Mpondo BC. Magnitude and correlates of moderate to severe anemia among adult HIV patients receiving first line HAART in Northwestern Tanzania: a cross sectional clinic based study. Pan Afr Med J. 2016;23(26):26.

33. Manaye Y, Asrat A, Mengesha EW. Time to Development of Anemia and Predictors among HIV-Infected Patients Initiating ART at Felege Hiwot Referral Hospital, Northwest Ethiopia: A Retrospective Follow-Up Study. BioMed Res Int. 2020;2020(7901241):7901241.

34. Silva RA, Costa RH, Nelson AR, Duarte FH, Prado NC, Rodrigues EH. Predictive factors for the nursing diagnoses in people living with acquired immune deficiency syndrome. Rev Lat Am Enfermagem. 2016;24:e2712. 